\title{
Acidentes de trabalho em ambiente hospitalar e riscos ocupacionais para os profissionais de enfermagem
}

\author{
Work accidents in a hospital setting and occupational risks \\ for nursing professionals
}

\author{
Iara Aparecida de Oliveira Sêcco ${ }^{1}$; Paulo Roberto Gutierrez ${ }^{2}$; Tiemi Matsuo ${ }^{3}$
}

\section{Resumo}

Os trabalhadores de enfermagem que atuam em unidades hospitalares estão expostos a muitos riscos ocupacionais que culminam com a ocorrência de acidentes de trabalho de variadas naturezas. Este estudo apresenta revisão de literatura a respeito dos acidentes de trabalho que acometem os referidos profissionais; caracteristicamente, os trabalhadores de enfermagem dedicam-se ao cuidado dos pacientes e necessitam que seja dedicado igual cuidado com a própria saúde. Estratégias preventivas apresentam-se como desafio para administradores e trabalhadores, onde o maior ganho está na promoção da saúde destes profissionais.

Palavras-chave: Acidentes de Trabalho, enfermagem, riscos ocupacionais.

\begin{abstract}
Nursing professionals working in hospital units are exposed to many occupational risks which may mean work accidents of most varied types. This study is a literature review on these types of damages caused to those professionals. Characteristically, these professionals devote themselves to the care of patients and are required to have identical care given to their own health. Preventive strategies are a challenge for managers and workers, whose greatest gain is in the promotion of these professionals' health.
\end{abstract}

Key-words: Work accidents, nursing, occupational risks.

\section{Introdução}

Os trabalhadores de enfermagem, inseridos na atividade de prestação de serviços de saúde, executam atividades que requerem grande proximidade física com o paciente pela característica do cuidar em enfermagem, bem como pela utilização e manuseio de materiais e equipamentos. A assistência de enfermagem favorece, por isso, a ocorrência de acidentes de trabalho (AT) e, de maneira saliente, daqueles acidentes advindos da exposição a materiais

\footnotetext{
1 Docente de Enfermagem da Universidade Norte do Paraná, Mestre em Saúde Coletiva e Enfermeira da Assessoria de Enfermagem no Planejamento e Controle do Hospital Universitário Regional do Norte do Paraná Avenida Arthur Thomas, 1355, Casa 2 CEP 86065-000 Jardim Bandeirantes - Londrina PR - Fone: (43) 3284005 - E-mail: nhsecco@ uol.com.br

2 Docente do Departamento de Saúde Coletiva do Centro de Ciências da Saúde da Universidade Estadual de Londrina, Doutor em Ciências da Saúde.

3 Docente do Departamento de Matemática Aplicada do Centro de Ciências Exatas da Universidade Estadual de Londrina, Doutora em Estatística.
} 
Sêcco, I. A. de O . et al.

biológicos como sangue e outros fluidos corporais. Este artigo apresenta revisão de literatura a respeito do tema Acidentes de Trabalho destacando os riscos ocupacionais e os aspectos inerentes ao processo de trabalho destes profissionais.

\section{Acidentes de Trabalho (ATs) e os Profissionais de Enfermagem}

Os ATs apresentam-se como a concretização dos agravos à saúde dos trabalhadores em decorrência da atividade produtiva, ao receberem interferências do que diz respeito à aspectos inerentes à própria pessoa, do ponto de vista físico ou psíquico, bem como do contexto social, econômico, político e da própria existência (BARBOSA, 1989; SILVA, 1996). Eles decorrem da ruptura na relação entre o trabalhador e as condições e/ou ambiente de trabalho, os quais interferem no seu processo saúde-doença (MENDES, 1995).

Gelbcke (1991), em estudos a respeito do trabalho dos profissionais de enfermagem, afirma que "o processo saúde-doença da classe trabalhadora tem como condicionantes básicos: as condições gerais de vida, as relações de trabalho e o próprio processo de trabalho".

Considerando-se que os ATs podem ocorrer de maneira abrupta ou insidiosa, em decorrência do exercício profissional e do modo de viver destes trabalhadores, faz-se necessária atenção de todos os envolvidos nas relações de trabalho, quer empregados quer empregadores.

Segundo a Lei $n^{\circ} .8 .213$, de 24 de julho de 1991, alterada pelo Decreto ${ }^{\circ}$. 611 , de 21 de julho de 1992 , no artigo $19^{\circ}$, reiterada ainda pela Lei Estadual do Paraná $n^{\circ} .10 .692$ de 27 de dezembro de 1993, Acidente do Trabalho é aquele que ocorre pelo exercício do trabalho, a serviço da empresa ou, ainda, pelo serviço de trabalho de segurados especiais, provocando lesão corporal ou perturbação funcional que cause a morte, a perda ou redução da capacidade para o trabalho, permanente ou temporária (BRASIL, 1992).
São considerados, ainda, como ATs os acidentes de trajeto, as doenças profissionais e as doenças do trabalho.

Os profissionais da equipe de enfermagem, especialmente aqueles da assistência hospitalar, estão expostos à ocorrência de ATs de variadas naturezas, resultantes dos processos de trabalho desenvolvidos e, por conseguinte, dos processos de produção de serviços que envolvem a promoção e preservação da saúde dos usuários dos serviços de saúde. Salienta-se, entre os agravantes que predispõem a estas ocorrências, a proximidade física necessária na prestação da assistência de enfermagem, bem como os processos de trabalho indiretos envolvidos na prestação desta mesma assistência.

Historicamente, os profissionais de enfermagem estão ligados e preparados para o cuidado, para a assistência integral ao paciente.

Entretanto, precisam receber a mesma contrapartida, a mesma atenção com a sua própria saúde. Mauro et al. (1976) referem que o pessoal de enfermagem tem a função de contribuir para a preservação da vida e saúde das pessoas, embora não consigam resolver os problemas relativos à sua própria saúde.

Afinal, "quem cuida de quem cuida?", "quem cuida daquele que tem como essência o cuidado na sua profissão?" (COSTENARO; LACERDA, 2001).

Estas indagações têm-se tornado uma constante diante das discussões a respeito dos trabalhadores de enfermagem, das suas condições de trabalho e de existência, da maneira como o trabalho é organizado e desenvolvido. A visão idealizada da profissão de enfermagem se contrapõe à dura realidade de um trabalho mal-remunerado, com excessiva carga de atividades, relacionada, na maioria das vezes, com a doença e com a morte (AQUINO et al., 1993; ROBAZZI; MARZIALE, 2000).

É importante considerar que o ambiente hospitalar, que absorve grande número destes profissionais, mostra-se reconhecidamente insalubre por agrupar 
portadores de diversas enfermidades infecciosas, além de viabilizar procedimentos que oferecem riscos profissionais diversos para os trabalhadores que atuam nestas Instituições (PRADO et al.,1999).

Pitta (1999), estudando relações entre o sofrimento psíquico e o trabalho hospitalar, mostra que os hospitais têm sido espaços de concentração de trabalhadores de diversas áreas, desde médicos, enfermeiros, auxiliares de enfermagem, operadores de máquinas, entre muitos outros, que se inter-relacionam com os usuários dos serviços. Também relata que, nos hospitais, o avanço tecnológico e científico tem apresentado desenvolvimento amplo, querendo refletir o alcance de maior nível de desenvolvimento da sociedade.

Bulhões (1994) ressalta que, uma vez que o hospital se apresenta como principal meio ambiente de trabalho para os profissionais de enfermagem, fazse necessário considerar que o ambiente é nocivo e pode trazer consequiências graves, dada a exposição diária dos profissionais a este ambiente reconhecidamente insalubre.

A autora prossegue afirmando que os riscos profissionais estão associados, sobretudo, ao fato de que os profissionais de enfermagem representam o maior grupo da área de assistência à saúde, prestam assistência ininterrupta nas 24 horas do dia, são responsáveis pela execução de cerca de $60 \%$ das ações relacionadas ao atendimento de paciente/clientes, estão fisicamente mais próximos dos usuários, são majoritariamente do sexo feminino, possuem diversidade de formação profissional entre os trabalhadores da equipe.

Oliveira, Makaron e Morrone (1982) consideram o ambiente hospitalar como risco não só de acidentes decorrentes do contato com pacientes portadores de doenças infecciosas, mas também daqueles decorrentes do caráter industrial que têm essas Instituições, como o caso dos serviços que envolvem centrais de processamento e esterilização de materiais, cozinha, manutenção de equipamentos, zeladoria, laboratórios, lavanderia, entre outros.
Os ATs que acometem os profissionais de enfermagem derivam de complexas inter-relações e têm sido objeto de muitos estudos no âmbito da enfermagem, por meio da análise destas ocorrências não apenas de forma isolada, como evento particular, mas através da análise do contexto do trabalho e das condições de vida dos profissionais expostos a estes infortúnios.

A temática em torno da prevenção de ATs remete ao estudo dos riscos ocupacionais a que estão expostos os trabalhadores de enfermagem. Para Barbosa (1989), os ATs representam a efetivação dos riscos ocupacionais, tratando-se de fatores determinantes do processo saúde-doença destes trabalhadores e do desgaste de suas funções vitais, do ponto de vista físico, psíquico e social. A autora mostra a importância e a complexidade das conseqüências destes fatores de risco, de origem ergonômica, física, biológica ou química.

Com relação aos riscos biológicos, Barbosa (1989) discute a sua importância em razão da função reprodutora da mulher, uma vez que o maior contingente de trabalhadores dos hospitais é do sexo feminino. Prossegue ponderando que, entre os agentes infecciosos, os vírus são os que têm maior capacidade para desencadear malformações fetais, sem contar as bactérias que podem alterar a morfologia do feto através de seus processos inflamatórios. Também destaca os riscos biológicos da hepatite $\mathrm{B}$, a que os profissionais de saúde estão sobremaneira expostos.

O enfoque da prevenção dos ATs e minimização dos riscos ocupacionais do pessoal de enfermagem tem sido objeto de muitos estudos. É fato que existe um número variado de fatores que interagem de maneira a propiciar as ocorrências, também definidos como multicausalidade. Leser et al. (2000) afirmam que o "[...] processo complexo de alterações de graus de saúde é uma cadeia contínua de causas e efeitos e não o resultado de uma causa única ou específica".

Nesta mesma busca do estudo da multicausalidade que envolve os ATs, Benatti (1997) assevera que a verdadeira causa dos acidentes deve 
Sêcco, I. A. de O . et al.

ser buscada nas condições de trabalho e existência da classe trabalhadora. Enfatiza a necessidade de estudos que investiguem associações entre a ocorrência de ATs acometendo trabalhadores de enfermagem e os fatores de risco decorrentes destas mesmas condições de trabalho e vida.

Santos et al. (1989), pesquisando ATs típicos entre trabalhadores de enfermagem, encontraram dados que levantam a hipótese de que a desatenção e a desmotivação para o trabalho mais a fadiga foram responsáveis pela ocorrência dos acidentes, sugerindo novos estudos para estas possibilidades. Os autores relatam também que as variáveis econômicas e as ligadas ao exercício da profissão (categoria profissional, plantão, duplicidade de emprego, turno, unidade de trabalho, entre outros) foram mais relevantes que as variáveis biológicas (sexo, idade, gravidez, fase do ciclo menstrual) para a ocorrência dos acidentes. Acrescentam que os fatores econômicos e profissionais podem estar dissuadindo os indivíduos da realização do trabalho, e, em conseqüência, os indivíduos estariam cumprindo tarefas sem a atenção necessária para evitar o acidente.

Outro dado digno de ser salientado é o fato de que os ATs então diretamente ligados também à inserção social dos trabalhadores no contexto da atividade laboral, dentro da própria classe trabalhadora. Estudos mostram a diferença entre os acidentes que acometem enfermeiros, auxiliares e atendentes de enfermagem, pela especificidade das atividades desempenhadas pelos profissionais das diferentes categorias, ligadas às características dos processos de trabalho, e, em conseqüência, ao desgaste do trabalhador de enfermagem.

Para Rodriguez, Magalhães e Sales (1995), atualmente, no Brasil, têm sido feitos diversos questionamentos a respeito do exercício profissional da enfermagem e das diversas categorias que a compõem. A estrutura dos hospitais apontam para a divisão do trabalho por categorias; por conseguinte, a enfermagem é marcada nitidamente por esta divisão do trabalho na área assistencial.
Esses autores citam Mendes (1985) salientando que o enfermeiro se afasta da sua responsabilidade direta da assistência de enfermagem ao paciente, assim como da família e da comunidade, assumindo outras funções, como a administrativa. Com isso, sofre influência de muitos fatores intrínsecos ao seu campo de prática, dos quais ele não detém controle, distorcendo ainda mais as suas funções. Por essa razão, a assistência fica delegada aos demais componentes da equipe, com menor qualificação profissional.

Silva (1996) discute que esse desgaste, diferenciado de acordo com as categorias de profissionais, possivelmente expresse a exposição do trabalhador às cargas de trabalho específicas e de diferentes intensidades, "através da interação do trabalhador com o objeto, meios e instrumentos de trabalho e formas de organização e divisão do trabalho".

Sendo assim, os problemas de saúde que apareceram com maior frequiência entre as diferentes categorias de trabalhadores são evidenciados a partir da inserção social específica dos trabalhadores na dinâmica do trabalho e do próprio processo de trabalho.

Um indicador dessa afirmação é que, na referida pesquisa, os problemas ósteomusculares só se apresentaram entre os atendentes e auxiliares de enfermagem. Provavelmente, o resultado se deve ao fato destes últimos estarem mais expostos às cargas de trabalho fisiológicas e mecânicas, em razão da manipulação excessiva de peso, permanência em pé durante a jornada de trabalho e frequientes deslocamentos dentro da unidade de trabalho, tarefas diretamente ligadas ao "processo assistir/cuidar", conforme afirma a autora.

Outro fato digno de nota apresentado por Silva (1996) é que, do total de 20 grupos de tipos de morbidade (tais como os ferimentos perfurocortantes, as doenças infecciosas, as doenças infectocontagiosas, a enxaqueca e cefaléia, os problemas auditivos, os disturbios do sono, entre outros) pesquisados entre os trabalhadores de enfermagem, 13 deles apresentaram-se apenas nas categorias de auxiliares e 
atendentes de enfermagem, sugerindo, segundo a autora, maior "carga de trabalho e maior intensidade do trabalho sobre estes trabalhadores".

Também Alexandre e Benatti (1998), estudando ATs que afetam a coluna vertebral de trabalhadores de enfermagem, encontraram maior proporção entre os técnicos de enfermagem do que entre as demais categorias, reforçando que a qualificação profissional não é a tônica para a ocorrência dos acidentes de trabalho. Chamam a atenção, ainda, para o fato de que doenças osteomusculares podem surgir em decorrência de um acidente específico ou de maneira lenta não sendo facilmente reconhecida a relação com o trabalho, a qual, muitas vezes, é atribuída à idade e a fatores individuais ou hereditários.

Sendo assim, é possível indicar que, de acordo com a inserção do trabalhador de enfermagem na própria classe, maiores ou menores serão os riscos a que eles estarão expostos (SILVA, 1996).

\section{Considerações Finais}

É fato que o estudo dos ATs que acometem os profissionais de enfermagem requer a consideração de um arsenal de variáveis que dizem respeito ao grupo profissional, que está constituído por trabalhadores de diferentes categorias e diferentes inserções sociais, econômicas e culturais.

Contudo, "é necessário que os trabalhadores de Enfermagem redimensionem a sua vida profissional e aprendam a mostrar aos seus clientes a importância benéfica de uma boa atuação, através da prestação de cuidados de enfermagem cientificamente embasados, ao lado de um profissional provedor desses cuidados, essencialmente hígido e em estado de bem-estar no seu ambiente laboral" (ROBAZZI; MARZIALE 2000, p.333).

Em síntese, é relevante o problema dos ATs que envolvem a equipe de enfermagem. Entretanto, na busca de estratégias que previnam os trabalhadores desses infortúnios, há que se atentar para os processos de trabalho envolvidos no desenvolvimento dessas atividades e nas relações sociais em que estes se dão.

\section{Referências}

ALEXANDRE, N. M.; BENATTI, M. C. C. Acidentes de trabalho afetando a coluna vertebral: um estudo realizado com trabalhadores de enfermagem de um Hospital Universitário. Rev. Lat. Am. Enfermagem, São Paulo, v.6, n.2, p.65-72, abr.1998.

AQUINO, E. M. L. et al. Saúde e trabalho de mulheres profissionais de enfermagem em um hospital público de Salvador, Bahia. Rev. Bras. Enfermagem, Brasília, v.46, n.3/4, p.245-257, jul./dez.1993.

BARBOSA, A. Riscos ocupacionais em hospitais: um desafio aos profissionais da área de saúde ocupacional. 1989. 126f. Dissertação (Mestrado em Ciências da Enfermagem) - Faculdade de Enfermagem, Universidade Federal de Santa Catarina, Florianópolis.

BENATTI, M. C. C. Acidente do trabalho em um Hospital Universitário: um estudo sobre a ocorrência e os fatores de risco entre trabalhadores de enfermagem. 1997. $239 \mathrm{f}$. Tese (Doutorado em Enfermagem) - Escola de Enfermagem, Universidade de São Paulo, Ribeirão Preto.

BRASIL. Decreto nº 611, de 21 de julho de 1992. Dá nova redação ao regulamento dos Benefícios da Previdência Social. Lex: Coletânea de Legislação e Jurisprudência , São Paulo, v.6, p.488, jul./set. 1992.

BULHÕES, I. Riscos do trabalho em enfermagem. Rio de Janeiro: Ideas, 1994. 221p.

COSTENARO, R.G.S.; LACERDA, M.R. Quem cuida de quem cuida? Quem cuida do cuidador? Santa Maria: Centro Universitário Franciscano, 2001. 96p.

GELBCKE, F. L. Processo saúde-doença e processo de trabalho: a visão dos trabalhadores de enfermagem de um hospital escola. 1991. 266f. Dissertação (Mestrado em Enfermagem) Universidade do Rio de Janeiro, Rio de Janeiro.

LESER, W. et al. Elementos de epidemiologia geral. São Paulo: Atheneu, 2000. 177 p.

MAURO. M. Y. C. et al. Pesquisa em Enfermagem: fadiga e aspectos ergonômicos no trabalho de enfermagem. Rev. Bras. Enfermagem, Brasília, v.29, n.4, p.7-24, 1997.

MENDES, R. Aspectos históricos da patologia do trabalho. In: __ Patologia do trabalho. Rio de Janeiro: Atheneu, 1985. p.3-31.

MENDES, R. Aspectos conceituais da patologia do trabalho. In: Patologia do trabalho. Rio de Janeiro: Atheneu, 1995. p.34-47.

OLIVEIRA, M. G.; MAKARON, P. E.; MORRONE, L. C. Aspectos epidemiológicos dos acidentes de trabalho em um hospital geral. Rev. Bras. Saúde Ocup., Brasília, v.10, n.40, p.26-30, 1982. 
PITTA, A. M. F. Hospital: dor e morte como ofício. 3.ed. São Paulo: HUCITEC, 1999.

PRADO, M. A. et al. A equipe de saúde frente aos acidentes com material biológico. Nursing, São Paulo, v.2, n.19, p.22-24, dez. 1999.

ROBAZZI, M.L.C.C.; MARZIALE, M.H.P. Alguns problemas ocupacionais decorrentes do trabalho de enfermagem no Brasil. Rev. Bras. Enfermagem, Brasília, v.52, n.3, p.331-338, jul./set. 2000.

RODRIGUES, A. B. ; MAGALHÃES, M. B. B. ; SALES, S. M. M. A questão do vírus da imunodeficiência humana e auto proteção no trabalho. Rev. Bras. Enfermagem, Brasília, v.48, n.3, p. 272-285, jul./set. 1995.
SANTOS, W. D. F. et al. Acidentes típicos de trabalho em pessoal de enfermagem: fatores associados. Rev. Bras. Saúde Ocup., Brasília, v.17, n.68, p.38-42, out./dez. 1989.

SILVA, V. E. F. O desgaste do trabalhador de enfermagem: estudo da relação trabalho de enfermagem e saúde do trabalhador. 1996; 289f. Tese (Doutorado em Enfermagem) - Escola de Enfermagem, Universidade de São Paulo, São Paulo. 